\title{
Search for a high mass $Z \gamma$ resonance using the ATLAS detector
}

\section{Giovanni Marchiori*}

on behalf of the ATLAS Collaboration

LPNHE Paris

E-mail: giovanni.marchiorialpnhe.in2p3.fr

Searches are presented for high mass resonances, not predicted by the Standard Model, decaying to a $Z$ boson and a photon. The searches are performed using up to $13.3 \mathrm{fb}^{-1}$ of $\sqrt{s}=13 \mathrm{TeV}$ proton-proton collision data collected with the ATLAS detector at the Large Hadron Collider. Both leptonic and hadronic decays of the $Z$ boson are considered. No significant excess with respect to the expected background is observed in the data in the whole mass range investigated, and upper limits are set on the production cross section times decay branching ratio to $Z \gamma$ of a narrow scalar boson.

38th International Conference on High Energy Physics 3-10 August 2016

Chicago, USA

${ }^{*}$ Speaker. 


\section{Introduction}

Many extensions of the Standard Model (SM) predict new massive bosons, generically denoted here as $X$, arising for instance from an extension of the Higgs sector or from additional gauge fields. Most of such models predict $X$ boson decays to final states containing $W$ or $Z$ bosons or photons.

In this presentation, the results of two searches for $X \rightarrow Z \gamma$ resonances with a narrow intrinsic width produced in proton-proton ( $p p$ ) collisions at a centre-of-mass energy $\sqrt{s}$ of $13 \mathrm{TeV}$ are shown [1,2]. The data were collected with the ATLAS detector [3] at the Large Hadron Collider (LHC). Previous searches for $Z \gamma$ decays of non-SM bosons produced in $\sqrt{s}=1.96 \mathrm{TeV} p \bar{p}$ collisions at the Tevatron [4] or in $\sqrt{s}=7$ and $8 \mathrm{TeV} p p$ collisions at the LHC $[5,6]$ yielded no evidence of such new particles.

The first search presented here [1] is based on the full $p p$ collision dataset collected in 2015, corresponding to an integrated luminosity of $3.2 \mathrm{fb}^{-1}$, and considers both decays of the $Z$ boson to a lepton-antilepton pair $\ell^{+} \ell^{-},{ }^{1} \ell=e, \mu$ (with a branching ratio $B R \approx 6.7 \%$ ), or to a quark-antiquark pair $q \bar{q}(B R \approx 70 \%)$. The second analysis [2] uses an additional $10.1 \mathrm{fb}^{-1}$ of $p p$ collisions, collected up to July 2016, to increase the sensitivity of the $X \rightarrow Z(\rightarrow \ell \ell) \gamma$ search and extend its mass reach. Similar studies have been performed recently by the CMS collaboration, too. [7, 8, 9].

In the following, the search based on the selection of $\ell \ell \gamma$ final states is also referred to as the leptonic analysis, while the search based on the selection of the $q \bar{q} \gamma$ final state is also referred to as the hadronic analysis. The two analyses are complementary: the hadronic one is characterised by larger backgrounds and worse resolution on the final state invariant mass, but the ten times larger $B R$ leads to a larger expected signal and better sensitivity than the leptonic analysis at high mass.

\section{Analysis strategy}

The leptonic analysis uses events collected with lepton triggers to search for $X$ bosons with a mass $\left(m_{X}\right)$ between $250 \mathrm{GeV}$ and $1.5 \mathrm{TeV}$ in the 2015 dataset, and in the range $250 \mathrm{GeV}-2.4 \mathrm{TeV}$ in the larger 2015+2016 dataset. On the other hand, the hadronic analysis uses events selected with a single photon trigger and targets $X$ bosons with masses between $700 \mathrm{GeV}$ and $2.75 \mathrm{TeV}$. Due to the large value of $m_{X}$ and thus to the large Lorentz boost of the $Z$ boson, the particles produced by the hadronisation of the $q \bar{q}$ pair from the $Z$ boson decay are sufficiently close in space to make it impractical to resolve the two separate jets of particles from the two quarks. The hadronic analysis thus reconstructs the $Z$ boson four-momentum from that of a large-radius $(R=1)$ "jet" $J$, built using the anti- $k_{t}$ algorithm from topological clusters of energy deposits in the ATLAS calorimeter.

All the analyses presented here search for a localized excess in the invariant mass distribution $m_{\text {inv }}$ of the reconstructed final state over a smooth background from SM processes. The signal yield and its statistical significance are determined by means of a profile-likelihood-ratio method [10], in which the data $m_{\text {inv }}$ distribution is fit with the sum of a function describing the signal $(S)$, with parameters determined from simulated signal samples, and a function characterising the background $(B)$, with parameters floating in the fit. Systematic uncertainties are incorporated in the likelihood through "constraint terms", and "profiled" by the fit. They arise from uncertainties on the integrated luminosity, on the lepton, photon and jet reconstruction and selection efficiencies and on

\footnotetext{
${ }^{1}$ In the following, $\ell^{+} \ell^{-}$final states are referred to as $\ell \ell$ for simplicity
} 
their energy scale and resolution, and on the possible bias ("spurious signal") on the fitted signal yield due to the choice of the background $m_{\mathrm{inv}}$ function. Their impact on the final result is at most $5-10 \%$ in the hadronic analysis and $1-2 \%$ in the leptonic analysis.

The simulated signal samples, which are also used to determine the selection efficiency needed to translate the signal yield into a cross section measurement, assume a scalar boson $X$ with intrinsic width of $\approx 4 \mathrm{MeV}$, much smaller than the experimental resolution, produced in gluon fusion events. The samples are generated with POWHEG-BOX interfaced to PYTHIA 8 and passed through a full simulation of the ATLAS detector response.

In the leptonic analysis, the main background arises from continuum production of a $Z$ boson in association with a photon, or, to a lesser extent, with a hadronic jet misidentified as a photon. In the hadronic analysis, the background is dominated by non-resonant SM production of $\gamma+$ jet events, with smaller contributions from dijet events with a jet misidentified as a photon, and from SM $V+\gamma$ events $(V=W, Z)$. The dominant background sources $(Z \gamma$ and $\gamma+$ jet for the leptonic and hadronic analyses, respectively) contribute to more than $90 \%$ of the total background; their fraction is estimated in situ using control samples of photon candidates failing either or both the identification and isolation requirements applied to the signal candidates as described later. The background composition is only used in ancillary studies to estimate the spurious signal.

\section{Search for $Z \gamma$ resonances $(Z \rightarrow \ell \ell$ and $Z \rightarrow q \bar{q})$ with $3.2 \mathbf{f b}^{-1}$ of $p p$ collisions}

In the leptonic analysis, events are selected by requiring two same-flavour, opposite-sign charged leptons (either electrons or muons) with transverse momentum $p_{\mathrm{T}}>10 \mathrm{GeV}$ and invariant mass of the pair within $\pm 15 \mathrm{GeV}$ of the $Z$ boson mass. Both leptons must satisfy identification criteria based on information from the inner charged particle tracker, the electromagnetic calorimeter or the outer muon spectrometer. To suppress fake candidates from hadron decays, the leptons are required to be isolated from significant activity in the adjacent calorimeter cells and in nearby regions of the tracker. Photons passing identification requirements, based on the longitudinal and lateral profile of the induced shower in the electromagnetic calorimeter and on the leakage in the hadronic calorimeter downstream, and satisfying isolation criteria, are retained. The three-body invariant mass $m_{\ell \ell \gamma}$ is computed from the lepton and photon four-momenta using a $Z$ boson-mass-constrained kinematic fit and assuming the photon to originate from the same space point as the two leptons. These corrections improve the $m_{\ell \ell \gamma}$ resolution by up to $20 \%$. Only events with $m_{\ell \ell \gamma}>200 \mathrm{GeV}$ and $p_{\mathrm{T}}^{\gamma}>0.3 m_{\ell \ell \gamma}$ are kept; the latter requirement is particular effective in suppressing continuum $Z \gamma$ events while keeping a high efficiency for decays of a scalar boson $X$. In case of multiple $\ell \ell \gamma$ candidates selected in the same event, only the one corresponding to the higher- $p_{\mathrm{T}}$ photon and the lepton pair with mass closer to that of the $Z$ boson is retained.

In the hadronic analysis, photons are kept if $p_{\mathrm{T}}^{\gamma}>250 \mathrm{GeV}$ and if they pass the same identification and isolation criteria as in the leptonic analysis. Large-radius jets $J$ are "trimmed" by removing subjets (reconstructed from the initial jet constituents using a $k_{\perp}$ algorithm with radius parameter $R=0.2$ ) with $p_{\mathrm{T}}$ less than $5 \%$ of the $p_{\mathrm{T}}$ of the original jet. The jets $J$ are kept if $p_{\mathrm{T}}^{J}>200 \mathrm{GeV}$, if their mass $m_{J}$ is in the range $80-110 \mathrm{GeV}$, and if a "substructure" discriminant $D_{2}$, computed from the energy correlation functions of its constituents, favours the "two-prong" hypothesis (i.e. two nearby quarks from the $Z$ boson decay) over the "one-prong" hypothesis (i.e. the hadronisation of 
a sufficiently isolated hard gluon or quark). Only events with $m_{J \gamma}>640 \mathrm{GeV}$ are kept. In events with multiple $J \gamma$ candidates, only the one corresponding to the higher- $p_{\mathrm{T}}$ photon and jet is retained.

After the selection, in the data there are $382 Z(\rightarrow \ell \ell) \gamma$ candidates and $534 Z(\rightarrow J) \gamma$ candidates. The signal efficiency is estimated to range between $28 \%$ at $m_{X}=250 \mathrm{GeV}$ and $43 \%$ at $m_{X}=1.5$ $\mathrm{TeV}$ for the leptonic analysis and between $10 \%$ at $m_{X}=700 \mathrm{GeV}$ and $15 \%$ at $m_{X}=2.75 \mathrm{TeV}$ for the hadronic analysis. The signal and background models used to fit the data are a Gaussian with power-law tails and a function of the type $N\left(1-x^{k}\right)^{p_{1}} x^{p_{2}}\left(x=m_{\text {inv }} / \sqrt{s}, k=1\right.$ or $\left.1 / 3\right)$, respectively. The signal $m_{\text {inv }}$ (i.e. $m_{\ell \ell \gamma}$ or $m_{J \gamma}$ ) resolution is approximately $1 \%$ for the leptonic analysis and decreases from $3 \%$ to $1.7 \%$, for $m_{X}$ increasing from $700 \mathrm{GeV}$ to $2.75 \mathrm{TeV}$, for the hadronic analysis. The background function is chosen as the model with the minimum number of free parameters among those that give an adequate description of the data in the $S+B$ fit and that are expected, from fits to high-statistics background control samples, to lead to negligible biases on the fitted signal yield compared to its statistical uncertainty from the fit.

The invariant mass distributions of the selected candidates and the results of background-only fits are shown in Fig. 1. A good agreement between the data and the fit is observed. Deviations from the background-only hypothesis have at most a local statistical significance of $2.0 \sigma$.

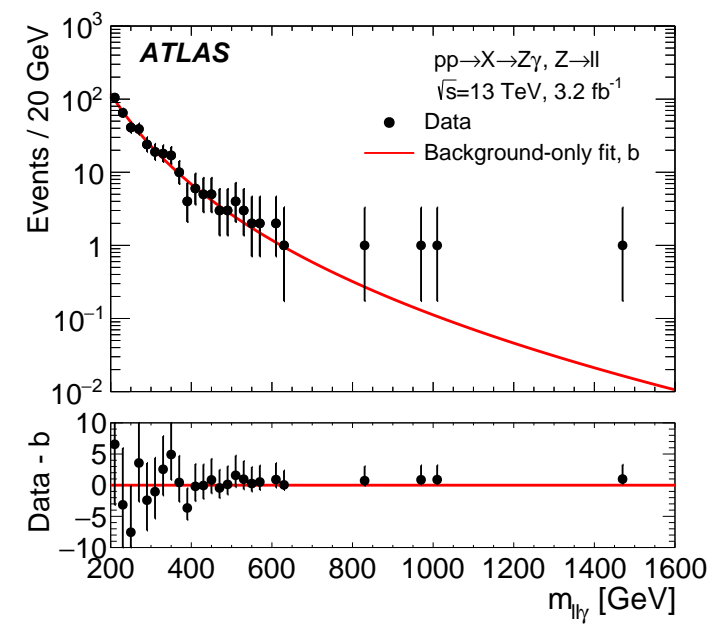

(a)

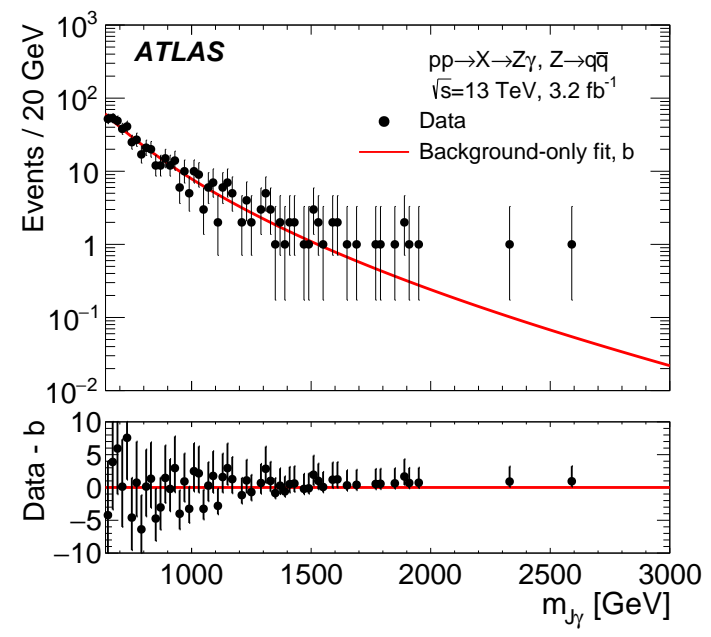

(b)

Figure 1: Distribution of the reconstructed $Z \gamma$ invariant mass in events in which the $Z$ boson decays to (a) electron or muon pairs, or (b) to hadrons reconstructed as a single, large-radius jet, in $3.2 \mathrm{fb}^{-1}$ of $p p$ collisions collected in 2015 [1]. The solid lines show the results of background-only fits to the data. In the bottom panels the residuals of the data points with respect to the fit are shown.

A modified frequentist method $\left(C L_{S}\right)$, with the asymptotic approximation to the test statistic distribution, is used to set $95 \%$ confidence-level (CL) limits on the production cross section times decay branching ratio to $Z \gamma$ of a narrow scalar boson $X, \sigma(p p \rightarrow X) \times B R(X \rightarrow Z \gamma)$. In the region $700 \mathrm{GeV}<m_{X}<1.5 \mathrm{TeV}$, tested by both the leptonic and the hadronic analysis, the results are combined, thus improving over the single results by up to $40 \%$. The final limits are shown in Fig. 2. The observed limits range between $295 \mathrm{fb}$ for $m_{X}=340 \mathrm{GeV}$ and $8.2 \mathrm{fb}$ for $m_{X}=2.15 \mathrm{TeV}$, while the expected limits range between $230 \mathrm{fb}$ for $m_{X}=250 \mathrm{GeV}$ and $10 \mathrm{fb}$ for $m_{X}=2.75 \mathrm{TeV}$. 


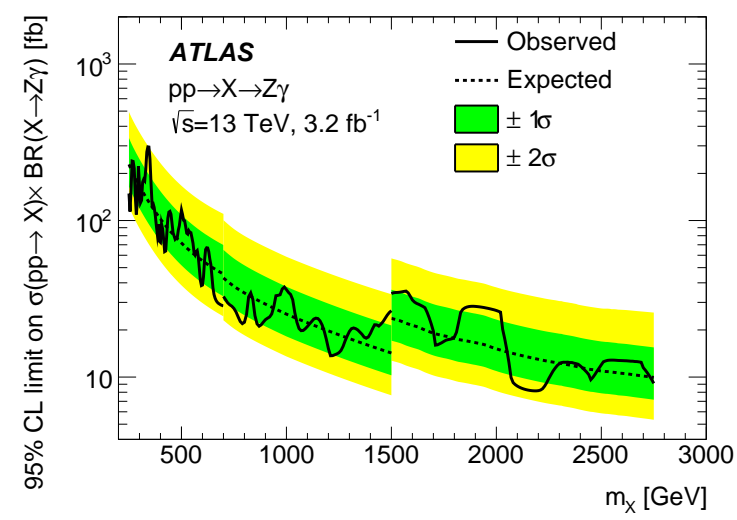

Figure 2: Observed (solid lines) and median expected (dashed lines) 95\% CL limits on the product of the production cross section times the branching ratio of a narrow scalar boson $X$ decaying to $Z \gamma$ as a function of its mass $m_{X}$, using $3.2 \mathrm{fb}^{-1}$ of $p p$ collisions collected in 2015 [1]. The green and yellow solid bands correspond to the $\pm 1 \sigma$ and $\pm 2 \sigma$ intervals for the expected upper limit respectively. For $250 \mathrm{GeV}<m_{X}<$ $700 \mathrm{GeV}$ and $1.5 \mathrm{TeV}<m_{X}<2.75 \mathrm{TeV}$ the limits are obtained from the leptonic and hadronic analyses respectively, while for $700 \mathrm{GeV}<m_{X}<1.5 \mathrm{TeV}$ they are obtained from the combination of the two analyses.

\section{Search for $Z \gamma$ resonances $(Z \rightarrow \ell \ell)$ with $13.3 \mathbf{f b}^{-1}$ of $p p$ collisions}

The leptonic analysis has been performed on the $\sqrt{s}=13 \mathrm{TeV} p p$ dataset collected until July 2016. In addition to the four times larger dataset, which allows the exploration of a wider mass interval, this analysis differs from the previous one by a reoptimisation of the reconstruction and selection criteria for the larger pileup of the 2016 data taking and for the introduction of separate event categories based on the lepton flavours, to exploit the different signal $m_{\ell \ell \gamma}$ resolution.

After the selection, in the data there are $306 Z(\rightarrow e e) \gamma$ candidates and $485 Z(\rightarrow \mu \mu) \gamma$ candidates. The invariant mass of the largest- $m_{\ell \ell \gamma}$ candidate is $1.5 \mathrm{TeV}(1.3 \mathrm{TeV})$ for $\ell=e(\mu)$. The efficiency of the selection increases from $22 \%$ (31\%) for $m_{X}=250 \mathrm{GeV}$ to $47 \%$ (46\%) for $m_{X}=2.4 \mathrm{TeV}$ for $\ell=e(\mu)$. The signal and background models used to fit the data are the same as in the leptonic analysis of the 2015 data, with updated signal parameters obtained from simulated signal samples that take into account the different data-taking conditions and selection criteria. Due to the different electron and muon momentum reconstruction techniques (either with a calorimeter or a tracking device), the $\ell \ell \gamma$ invariant mass resolution decreases (increases) from $1 \%$ (1\%) for $m_{X}=250 \mathrm{GeV}$ to $0.6 \%$ (1.4\%) for $m_{X}=2.4 \mathrm{TeV}$ for $\ell=e(\mu)$.

The invariant mass distribution of the selected candidates and the result of a background-only fit are shown in Fig. 3(a). A good agreement between the data and the fit is observed. Deviations from the background-only hypothesis have at most a local statistical significance of $2.2 \sigma$. Upper limits at $95 \% \mathrm{CL}$ are set on $\sigma(p p \rightarrow X) \times B R(X \rightarrow Z \gamma)$ using the $C L_{s}$ method. They are shown in Fig. 3(b). The observed limits range between $215 \mathrm{fb}$ for $m_{X}=270 \mathrm{GeV}$ and $5 \mathrm{fb}$ for $m_{X}=2.4 \mathrm{TeV}$, while the expected limits range between $103 \mathrm{fb}$ for $m_{X}=250 \mathrm{GeV}$ and $5 \mathrm{fb}$ for $m_{X}=2.4 \mathrm{TeV}$.

\section{Conclusion}

Searches for high mass resonances decaying to a $Z$ boson and a photon using up to $13.3 \mathrm{fb}^{-1}$ of 


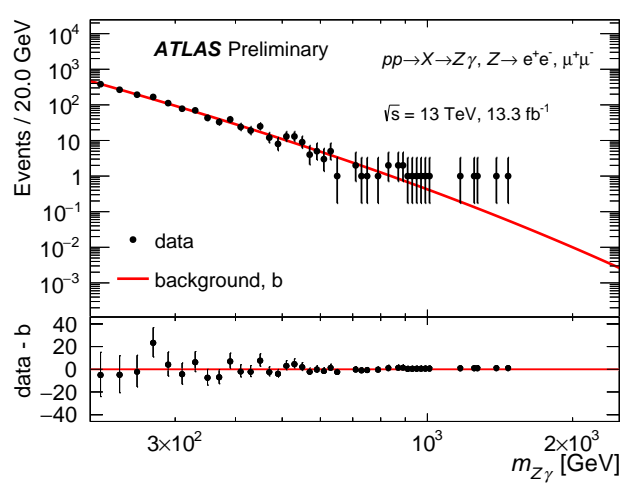

(a)

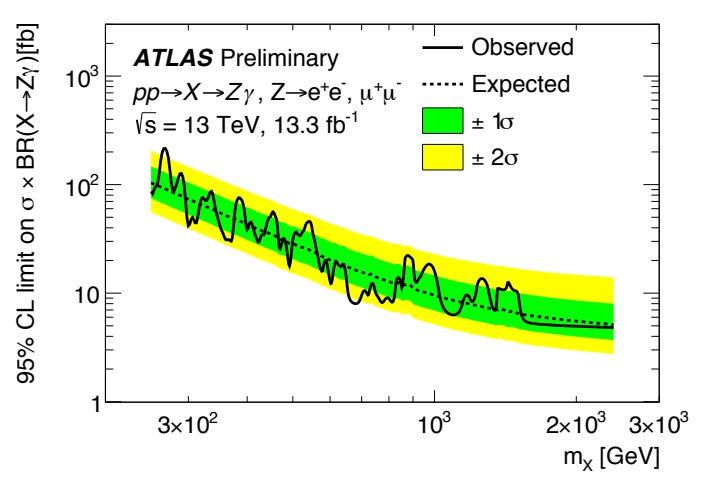

(b)

Figure 3: Results of the search of $X \rightarrow Z(\ell \ell) \gamma$ decays with $13.3 \mathrm{fb}^{-1}$ of $p p$ collisions collected in 2015 and 2016 [2]. Left: distribution of the $\ell \ell \gamma$ invariant mass of the selected candidates and result of a backgroundonly fit to the data. In the bottom panel the residuals of the data points with respect to the fit are shown. Right: observed and median expected 95\% CL limits on $\sigma(p p \rightarrow X) \times B R(X \rightarrow Z \gamma)$.

$\sqrt{s}=13 \mathrm{TeV} p p$ collision data collected with the ATLAS detector at the LHC found no significant signal. Upper limits on the production cross section times decay branching ratio to $Z \gamma$ of a narrow scalar boson are set between $5 \mathrm{fb}$ and $215 \mathrm{fb}$ for $m_{X}$ between $250 \mathrm{GeV}$ and $2.75 \mathrm{TeV}$.

\section{References}

[1] ATLAS Collaboration, "Search for heavy resonances decaying to a $Z$ boson and a photon in $p p$ collisions at $\sqrt{s}=13 \mathrm{TeV}$ with the ATLAS detector," arXiv:1607.06363 [hep-ex].

[2] ATLAS Collaboration, "Search for heavy resonances decaying to a $Z$ boson and a photon in $p p$ collisions at $\sqrt{s}=13 \mathrm{TeV}$ with the ATLAS detector," ATLAS-CONF-2016-044.

[3] ATLAS Collaboration, "The ATLAS Experiment at the CERN Large Hadron Collider," JINST 3 (2008) S08003.

[4] V. M. Abazov et al. [D0 Collaboration], "Search for a scalar or vector particle decaying into $Z \gamma$ in $p \bar{p}$ collisions at $\sqrt{s}=1.96$ TeV," Phys. Lett. B 671 (2009) 349 [arXiv:0806.0611 [hep-ex]].

[5] ATLAS Collaboration, "Measurements of $W \gamma$ and $Z \gamma$ production in $p p$ collisions at $\sqrt{s}=7 \mathrm{TeV}$ with the ATLAS detector at the LHC," Phys. Rev. D 87 (2013) 112003 [arXiv:1302.1283 [hep-ex]].

[6] ATLAS Collaboration, "Search for new resonances in $W \gamma$ and $Z \gamma$ final states in $p p$ collisions at $\sqrt{s}=8 \mathrm{TeV}$ with the ATLAS detector," Phys. Lett. B 738 (2014) 428 [arXiv:1407.8150 [hep-ex]].

[7] CMS Collaboration, "Search for high-mass Z-gamma resonances in $e^{+} e^{-} \gamma$ and $\mu^{+} \mu^{-} \gamma$ final states in proton-proton collisions at $\sqrt{s}=8$ and $13 \mathrm{TeV}$," arXiv:1610.02960 [hep-ex].

[8] CMS Collaboration, "Search for high-mass resonances in $Z \gamma \rightarrow e^{+} e^{-} \gamma / \mu^{+} \mu^{-} \gamma$ final states in proton-proton collisions at $\sqrt{s}=13 \mathrm{TeV}$," CMS-PAS-EXO-16-034.

[9] CMS Collaboration, "Search for high-mass resonances in $Z(\mathrm{q} \overline{\mathrm{q}}) \gamma$ final state in $p p$ collisions at $\sqrt{s}=13 \mathrm{TeV}$ with $12.9 \mathrm{fb}^{-1}$," CMS-PAS-EXO-16-035.

[10] G. Cowan, K. Cranmer, E. Gross and O. Vitells, "Asymptotic formulae for likelihood-based tests of new physics," Eur. Phys. J. C 71 (2011) 1554 [arXiv:1007.1727 [physics.data-an]]. 